\title{
LE TRAVAIL DES LIMITES DANS LE PROCESSUS D'ÉCRITURE. EXPLORATION DANS LA CRÉATION LITTÉRAIRE ET EN ATELIER D’ÉCRITURE
}

\author{
Olivia Lempen, Pascal Roman
}

\section{ERES | « Cliniques méditerranéennes »}

2017/2 n 96 | pages 107 à 121

ISSN 0762-7491

ISBN 9782749256474

Article disponible en ligne à l'adresse :

https://www.cairn.info/revue-cliniques-mediterraneennes-2017-2-page-107.htm

\section{Pour citer cet article :}

Olivia Lempen, Pascal Roman« Le travail des limites dans le processus d'écriture. Exploration dans la création littéraire et en atelier d'écriture », Cliniques méditerranéennes 2017/2 ( $\mathrm{n}^{\circ}$ 96), p. 107-121.

DOI 10.3917/cm.096.0107

Distribution électronique Cairn.info pour ERES.

(C) ERES. Tous droits réservés pour tous pays.

La reproduction ou représentation de cet article, notamment par photocopie, n'est autorisée que dans les limites des conditions générales d'utilisation du site ou, le cas échéant, des conditions générales de la licence souscrite par votre établissement. Toute autre reproduction ou représentation, en tout ou partie, sous quelque forme et de quelque manière que ce soit, est interdite sauf accord préalable et écrit de l'éditeur, en dehors des cas prévus par la législation en vigueur en France. Il est précisé que son stockage dans une base de données est également interdit. 


\title{
Olivia Lempen, Pascal Roman
}

\author{
Le travail des limites \\ dans le processus d'écriture. \\ Exploration dans la création littéraire \\ et en atelier d'écriture
}

Depuis les premières études de Freud touchant à la création artistique et à la sublimation, l'hypothèse de différents types de processus de création, qui n'aboutissent pas tous à l'émergence d'une œuvre créatrice de sens, a été développée par différents auteurs (Chouvier, 1998 ; Roussillon, 1998). Dans ce sens, la création peut s'inscrire dans des mécanismes relevant du clivage ou de la répétition, sans assurer nécessairement l'accomplissement de l'activité représentative. Elle peut exercer des fonctions psychiques relevant tout autant de l'élaboration que de la destruction, selon des modalités complexes qui ne se résument pas à l'aboutissement ou à l'échec de la symbolisation. $C^{\prime}$ est à partir de cette complexité des chemins de la création que se sont déployées nos réflexions dans le cadre d'une recherche en psychologie clinique centrée sur l'écriture et ses spécificités comme expérience créatrice.

Nos élaborations ont pris forme à partir d'un regard croisé sur les expériences de création d'écrivains publiés d'une part, et sur le processus d'écriture de participants à un atelier d'écriture en milieu de soins d'autre part. Cette double perspective sur l'écriture nous a permis d'articuler l'étude

Olivia Lempen, psychologue, docteure en psychologie clinique, art-thérapeute, Institut de psychologie, Faculté des sciences sociales et politiques, université de Lausanne, Geopolis 1015 Lausanne, Suisse ; olivia@lempen.me

Pascal Roman, psychologue, psychothérapeute, professeur de psychologie clinique, psychopathologie et psychanalyse, LARPsyDIS, Institut de psychologie, Faculté des sciences sociales et politiques, université de Lausanne, Geopolis 1015 Lausanne, Suisse ; pascal.roman@unil.ch 
des processus dans deux situations de la vie psychique : la création littéraire, à laquelle le sujet a recours naturellement dans le cours de son existence, et l'écriture en groupe comme situation construite pour agir sur le déploiement des processus et de leur fonction psychique. Une première phase du travail a été essentiellement exploratoire et a consisté à étudier les expériences d'écriture de manière approfondie afin de détailler et de comprendre les processus, les enjeux et les fonctions pour chacune d'elle, à partir du contexte dans lequel elles ont pris place (écriture littéraire, atelier d'écriture). À partir de cette première phase, nous avons pu dégager la place centrale de la mise au travail, dans le processus de création, des enjeux de continuité/discontinuité psychique dans la rencontre avec les lecteurs-auditeurs des textes. Ces constats nous ont conduit à une seconde phase d'élaboration centrée sur les processus d'écriture, à partir des notions de «Moi-peau » (Anzieu, 1985) et $\mathrm{d}^{\prime}$ « enveloppes psychiques » (Anzieu, 1981). En partant de l'hypothèse selon laquelle le travail de l'écriture peut contribuer à la restauration d'un Moi-peau fragile ou fragilisé, en agissant à la fois sur le rétablissement des limites et des échanges entre dedans et dehors, nos analyses ont permis de mettre en évidence un certains nombre de détours, voire d'impasses, au fil de ce processus.

\section{REPÈRES THÉORIQUES}

Winnicott (1971) se réfère aux premiers temps de l'existence pour montrer de quelle manière la rencontre avec l'environnement colore le rapport du sujet à la créativité et à la création tout au long de son existence. Le processus en «trouvé/créé » qu'il décrit permet d'appréhender la fondation des assises narcissiques du sujet et de mettre l'accent sur la dimension essentielle des caractéristiques de la réponse de l'environnement premier, réponse qui permet au bébé de trouver dans la réalité externe ce qui peut le confirmer dans ses capacités de pouvoir créer dans la réalité interne. Le " miroir » parental opère aux différents niveaux sensoriels, par le biais des soins corporels et du mode de présence de l'environnement. Ainsi, les qualités, les conditions et le degré de cette correspondance entre ce qui vient du dedans et ce qui arrive du dehors s'avèrent tout à fait déterminants pour le déploiement des processus de liaison et, au-delà, de symbolisation des premières expériences. Janin (2005) qualifie les expériences restées en souffrance de liaison de "noyau traumatique de tout processus psychique ", noyau à la source de la tension qui pousse le sujet à chercher des moyens d'intégration, ou de destruction, des traces perceptives restées non symbolisées. L'enjeu des processus psychiques de la création se situe dans cette quête de traitement du noyau traumatique, dans la quête d'un reflet qui aurait manqué à l'origine. 
Anzieu (1981) propose une théorie des processus de la création qui constitue, pour les psychanalystes contemporains, un repère des plus importants pour la compréhension des enjeux psychiques de la création. Les étapes du « travail créateur » sont pensées dans une articulation avec le corps du créateur, corps pulsionnel et sensoriel projeté dans « ce corps métaphorique » de l'œuvre. Guillaumin (1980, 1983, 1998), quant à lui, décrit le processus du " retournement projectif de l'intérieur du corps dans la création littéraire » (Guillaumin, 1980, p. 227) et propose de considérer cette dernière comme une " extension topique de l'appareil psychique », une " exotopique ». Cadoux, dans une réflexion portant sur l'écriture et la psychose, prolonge les hypothèses de Guillaumin et développe l'idée selon laquelle l'écriture vient « au secours de l'appareil psychique » $(2013$, p. 32) : « [...] l'écriture viendrait suppléer à la défaillance de l'appareil psychique en "donnant un lieu" à certains éléments traumatiques qu'il ne peut élaborer en son sein. L'écriture fournirait une inscription externe à ce qui ne s'est pas inscrit à l'intérieur. Elle assurerait en quelque sorte, par sa technique propre, le travail de contention et d'élaboration que le psychisme n'a pu effectuer, l'écriture constituant l'ultime rempart du moi contre l'effondrement qui le menace » (Cadoux, 2013, p. 34).

Guillaumin (1980) se réfère à l'image de la peau et à ses caractéristiques sensorielles pour préciser les conditions du retournement projectif dans l'écriture. Les supports de la projection, tout à la fois considérés en termes de surface réelle et matérielle (la feuille, la toile, etc.) et en termes de supports à la symbolisation, présentent une ressemblance fonctionnelle avec une peau, ou un fragment de peau. Dans cette perspective, la peau, " enveloppe et conteneur de la vie intérieure de l'auteur »(Guillaumin, 1980, p. 257), se retourne comme un gant et devient le support de la projection, le support de l'imaginaire de l'auteur. Anzieu (1981) reprend lui aussi l'image de la peau pour établir un parallèle entre la toile du peintre (ou la page blanche de l'écrivain) et la notion de " peau commune ». Il souligne ainsi la fonction interface de la toile du peintre, qui se constitue comme surface de contact mais aussi comme limite : la régression et la prise de conscience des représentants psychiques qui en découlent déclencheraient alors la réactivation de l'illusion d'omnipotence et, au-delà, soutiendraient une entreprise de reconstitution, dans le projet de composer l'œuvre, d' « une peau mi-matérielle mi-psychique venant réparer l'effraction » (Anzieu, 1981, p. 102) à la source du noyau traumatique. Dans cette même lignée, Emmanuelli (2001) reprend l'image de la peau proposée par Anzieu, en soulignant la fonction de restauration narcissique qu'elle remplit, tandis que Roman (2007) envisage l'expérience créatrice dans son rapport à l'expérience du corps et précisément à 
l'expérience de la peau. Jacques et Lefebvre (2005), quant à eux, reprennent l'analogie d'Anzieu pour l'élargir aux différentes fonctions du Moi-peau. Finalement, Cadoux synthétise les hypothèses de Guillaumin dans un propos spécifique à l'écriture: «Cela veut dire au fond que l'écriture est à la fois une tentative pour se créer une autre mère (suffisamment bonne) et se fabriquer une autre peau plus satisfaisante. » (Cadoux, 2013, p. 82)

\section{LA RENCONTRE DU PROCESSUS DE CRÉATION DANS L'ÉCRITURE}

L'approche clinique de la création confronte à des processus complexes qui peinent à se laisser saisir de manière univoque. Chouvier et Brun soulignent que la démarche créatrice "résiste, par sa nature même » (2011, p. 7) à toute tentative d'établir une théorie unique des pratiques artistiques. C'est dans cette perspective que nous avons tenté de contribuer à la compréhension des processus de la création dans leurs aspects singuliers et « énigmatiques » (ibid.) à partir desquels chaque expérience de création peut venir éclairer la connaissance du fonctionnement psychique. Un premier volet de notre recherche (Lempen, 2014) a consisté en l'étude des processus de la création littéraire à partir de l'analyse d'entretiens semi-structurés avec six écrivains publiés (deux poètes et quatre romanciers), choisis de manière aléatoire dans la région de Lausanne, où l'étude a été réalisée. Les entretiens se sont déployés à partir de questions ouvertes sur l'expérience de création en lien avec une œuvre en particulier pour chaque écrivain, choisie par lui. Un second volet de la recherche, mené parallèlement, a consisté en l'étude des processus d'écriture dans un contexte de soin groupal, à partir d'observations réalisées pendant une année au sein d'un atelier d'écriture pour des adultes dans un centre de jour, mené par une thérapeute et une stagiaire. L'atelier d'écriture se présentait comme un groupe ouvert en institution ; son déroulement s'appuyait sur la dimension groupale, en mettant l'accent sur la lecture des textes et sur les impressions que celles-ci véhiculaient dans le groupe. Les processus d'écriture de deux des participants au groupe ont fait l'objet d'élaborations à partir de la méthode du cas unique (Widlöcher, 1999).

\section{LES LIMITES À L'ÉPREUVE DE LA MATIÈRE}

L'étude exploratoire des expériences de création littéraire a mis en évidence trois temps principaux du processus d'écriture : la mise à l'écriture ; le travail de la matière ; le partage avec les lecteurs. Dans les propos des écrivains, le défi de la composition de l'œuvre, du travail de la matière, s'est dessiné autour de la possibilité de créer une forme littéraire qui puisse porter au regard d'un autre, lecteur, les parts de la vie intérieure restées en 
souffrance de représentation. Leurs propos ont témoigné de leur volonté $\mathrm{d}^{\prime}$ " exister », " d'être vu » dans leur intériorité, " de se dire de manière authentique ", portés par l'espoir que les lecteurs puissent reconnaître ce qui n'a pu être reconnu auparavant par eux-mêmes. Plusieurs des écrivains interrogés ont mentionné, au sujet de l'engagement dans leur activité d'écriture, des " situations d'urgence », " quelque chose qui pousse » et face à quoi ils ne pouvaient " pas faire autrement " que de se mettre à écrire. L'écriture s'engage, ainsi, par la projection d'un excès d'excitations internes qui " poussent » de l'intérieur et viennent se déposer sur le support papier, allant dans le sens d'un mouvement d'extériorisation urgent pour la survie psychique. Cette matière première projetée sur le papier est qualifiée $\mathrm{d}^{\prime}$ « impure » et les écrivains soulignent que, dans cette première forme, « ces parts d'ombre » ne peuvent être données à voir.

Lors du travail de la matière, que l'on peut identifier comme le travail du style, l'enjeu de la continuité entre le dépôt des impuretés sur la surface papier et la présentation du texte à des lecteurs devient essentiel. La complexité de ce travail se noue autour du vécu d'une menace permanente d'intrusion, voire de destruction, qui plane sur la quête de subjectivité par l'écriture ; le risque serait là de trop montrer et trop dévoiler, et d'être débouté de ce nouveau lieu pour l'affirmation de l'identité par le retour mortifère des éléments impensables. L'un des romanciers interrogés a particulièrement bien témoigné de ce conflit entre son désir d'être reconnu et la menace d'intrusion que cette quête de reconnaissance comporte. Lorsqu'il évoque la parution de son premier roman il s'en réfère autant au « au plaisir de voir les gens le découvrir autrement » qu'au sentiment «d'avoir été violé dans son intimité, d'avoir été piétiné ». Ainsi, l'un des enjeux du travail de composition de l'œuvre se situe autour du couple d'opposés montrer/ cacher et du travail des limites qu'il implique pour restaurer le sentiment de continuité. L'analyse des expériences d'écriture nous a permis de mettre en évidence différents procédés pour tenter de mettre en forme le caractère impur de ce qui cherche à s'exprimer dans la création, en vue de la présentation à cet autre à qui le texte s'adressera, et qui constituera un potentiel miroir symbolisant.

\section{LA MOBILISATION DES ACCORDAGES}

L'écriture peut mobiliser la mémoire des " accordages affectifs » (Stern, 1985). En effet, la mobilisation gestuelle et graphique qu'implique l'écriture, ses sonorités et son rythme, sont apparues comme les trois modalités majeures permettant d'engager le processus de (ré)accordage et de mise en forme des expériences impensables. L'un des poètes que nous avons rencontrés 
raconte ce processus à partir de l'expérience d'écriture de son premier recueil de poèmes : " Je me suis mis à tenir une espèce de journal où j'écrivais un peu ce qui me passait par la tête. J'ai bien dû noircir des centaines et des centaines de pages. [...] J'ai des parents qui ont toujours posé problème pour moi, que j'ai dû porter constamment parce que ça n'allait pas entre eux. [...] J'ai été anémotionnel dans certaines situations extrêmes, sans émotion [...] dans le clivage total. [...]. Dans mon recueil, c'est une immense tristesse qui est là derrière, de quelque chose de non vécu. L'objectif c'est peut-être d'essayer de rattraper ce qu'on n'a pas vécu, tout en sachant que c'est parfaitement dérisoire, ce qu'on n'a pas vécu, on ne le revivra jamais [...]. Écrire, c'est quand même laisser quelque chose, une trace, noir sur blanc, c'est au moins de l'avoir dit. [...] Cette urgence pour l'écriture était liée à une situation d'urgence dans la vie où je devais faire face constamment. [...] Quand j'ai écrit ce recueil, c'était encore à la main. Il y a les ratures [...]. J'essaie de suivre visuellement en voyant les mots, ils ont un aspect physique les mots, c'est des signes aussi. [...] Tout s'efface et on se met à chasser les mots, en partant d'une image. Les mots véhiculent des images mentales, des tas de choses. Et ensuite on les récite intérieurement à voix haute, parce qu'il faut un rythme aussi, tout à coup, ça se met à avoir un rythme. »

Cadoux (2013) décrit la manière dont l'écriture convoque d'abord le registre grapho-moteur, avant de s'inscrire dans celui des significations. Ici, le poète dit avoir "noirci des centaines et des centaines de pages », il précise qu'il écrivait «à la main », et se rappelle « les ratures » qu'il voit, sous la forme d'une image visuelle de ses écrits. Le souvenir visuel des pages noircies se constitue come première restitution transmodale de la violence, portée par le geste premier de l'écriture. Puis le poète se réfère à la « récitation à voix haute » qui lui permet, porté par la sonorité des mots, de donner une première forme aux images qui surgissent et « qui se mettent à avoir un rythme ». Les expériences infantiles insoutenables restées clivées trouvent ici un lieu d'accueil suffisamment contenant où les premiers accordages peuvent se rejouer dans leurs modalités tactiles (gestuelles), rythmiques et sonores et permettre au sujet de se ressaisir des expériences pour s'engager sur la voie de la symbolisation. L'écrit se constitue comme premier miroir sensoriel accordé.

Après cette première mise en forme graphique, sonore et rythmique du noyau pulsionnel, le processus de création est marqué par la nécessité de protéger ce qui a pu être accueilli sur le papier en vue de sa présentation à l'environnement. Les écrivains se réfèrent tous à la notion de fiction qu'ils rapportent à différents procédés visant à protéger leur intimité : "masquer », " traduire des émotions », mais aussi « couvrir », " habiller » ou encore 
" éplucher » et « remplumer ». Ces procédés se mettent en place dans le prolongement de l'expérience de la relation à l'objet premier, guidés par la figure du public intérieur (M'Uzan, 1964) et la tension entre des mouvements de réparation et de destruction.

Le poète précédemment cité se réfère au processus de «transfiguration poétique » qui lui permet "d'explorer les territoires intérieurs » tout en proposant des «territoires de rencontre». Il mentionne notamment comment le langage poétique permet de "masquer l'intimité pour ne pas la rendre directement accessible ». L'écriture poétique lui permet de reconstituer "son territoire intérieur", d'explorer sa matière et ses limites, tout en le rendant « accessible » pour une rencontre avec le monde qui l'entoure : «Les mots laissent assez de latitude. [...] Ils permettent des tas d'acceptions pour s'ouvrir aux autres [...] La poésie est le seul lieu où l'on peut légitimement dire Je [...] en tant que poète j'ai le droit de dire Je, et si je dis Je, je dois le faire de la manière la plus authentique possible, même si ça semble inauthentique et artificiel d'utiliser des tournures poétiques [...] Mais je ne peux pas le dire autrement non plus, parce que je ne me situe pas à un niveau qui est celui du moi moïsme, du nombrilisme, ce n'est pas du tout ça. On essaie par ces mots de transfigurer le Je en quelque chose de plus universel, parce qu'au bout du compte il y aurait une expérience qui serait valable pour tout le monde. [...] La poésie m’a permis de devenir quelqu'un. »

L'écriture poétique contribuerait ainsi au rétablissement d'une enveloppe contenante, qui permet au poète de restaurer son sentiment de continuité mis à mal dès sa plus jeune enfance. Ses propos évoquent de quelle manière la poésie cherche à travailler sur les deux faces du narcissisme en permettant au Je de se dire de manière « authentique » tout en soignant la couche superficielle, que le poète qualifie par ailleurs d' « artifices », pour protéger l'intimité.

\section{LA SECONDE PEAU-POÈME}

Pour certains des participants à notre étude, la menace d'intrusion liée à la présentation au dehors de la création est si forte qu'il est nécessaire de recourir de manière prioritaire à un investissement de l'écriture dans sa fonction de protection. Nous proposons l'hypothèse selon laquelle, dans ce cas de figure, le travail de transformation et d'intégration de la matière mi-psychique mi-matérielle projetée sur le papier est suspendu pour favoriser la matérialisation scripturale d'une enveloppe externe de protection.

Dans le groupe, les enjeux du travail du style se donnent à voir dans une forme externalisée, dans un rapport presque immédiat au lecteur. 
Le dispositif de l'atelier d'écriture présente la spécificité d'une quasi-juxtaposition du travail de l'écriture et de la rencontre des lecteurs-auditeurs, le groupe, et il a, de ce fait, permis d'observer le temps de la négociation qui suit la projection sur le papier, entre ce qu'il faut garder, montrer ou cacher. En effet, la situation groupale soumet le sujet aux effets de régression et de menace identitaire qu'elle induit (Anzieu, 1978) et face à ce danger dans le groupe, l'écriture peut être investie de façon prioritaire dans sa fonction défensive, afin de protéger le sujet de la répétition d'une expérience de discontinuité lors de la rencontre avec l'autre. L'analyse du processus d'écriture de l'un des participants au groupe, Jonathan, en témoigne.

Jonathan a rejoint l'atelier d'écriture au début de la période d'étude et a adopté une présence régulière au sein de l'atelier. Les premières séances sont marquées par des mouvements de régression propre à la situation groupale. Ses poèmes privilégient la simultanéité des images plutôt que leur mise en récit et se déclinent parfois dans une forme indéfinie sans sujet, rappelant le temps de l'indifférenciation. Les contenus figurent un retour à l'origine et parfois une expression crue de la destructivité :

« Je me sens immobile

dans la case de la ville avec le sens du temps

sans la contrainte du mouvement retourner à l'état végétatif

s'échouer sur le récif

de son enfance oubliée [...]

Celui d'un temps révolu Immobile et absolu. »

«Soldat du levant

il rit en tuant

se moque de ses coups de sang

dynamite les étangs

abat les derniers fuyants

dans la fureur et le boucan

couche et fiche le camp

sans se soucier des sentiments

balaie le visage de ses parents

noie ses enfants dans le courant

il est comme le temps

intansigeant

violent. » 
Cette première phase dans son écriture donne lieu à une écriture des traces témoignant d'un rapport à la temporalité brouillé. Jonathan privilégie le rythme de l'écriture poétique par rapport à celui de l'écriture narrative, dans un mouvement qui vient rappeler les premiers accordages affectifs et la restauration de vécus de contenance, comme nous avons pu l'évoquer dans l'expérience du poète. Cependant, assez vite, les menaces d'anéantissement se font tout aussi présentes dans ses textes et viennent témoigner du potentiel traumatique de la situation groupale :

«[...] Le monstre fit perdurer pendant un temps

le désir de faire renaître les saveurs d'antan

mais au moment où l'homme prit conscience

de la nature de l'être et de son efficience

il prit peur et s'inventa une menace

pour légitimer la survie de sa race

c'est le prix à payer lorsqu' on prend pour demeure

une bâtisse dont les murs sont forgés dans la peur

destin cruel ou ironie du sort

son dernier refuge fut un lit de mort [...]»

À ce moment du parcours de Jonathan dans le groupe, l'esquisse d'une enveloppe rythmique, contenante des éprouvés, est mise à mal par les stimulations du groupe. À cette même période Jonathan se met à tracer des cadres graphiques aux quatre coins des ses textes dans une probable tentative de matérialiser visuellement un contenant, pour protéger l'espace scriptural qui figure les instants de plénitude et d'omnipotence. Les cadres visuels ne semblent toutefois pas suffire pour lutter contre les menaces d'intrusion et, lors d'une séance particulièrement agitée, Jonathan doit quitter le groupe. Lorsqu'il revient, après une absence de quelques séances, il continue à investir le rythme du poème mais aussi sa structure en vers réguliers, les strophes et les rimes. Ce surinvestissement de la structure et des rimes suscite un mouvement d'idéalisation de Jonathan par le groupe qui le qualifie dorénavant de « grand poète ».

Nous faisons l'hypothèse que Jonathan surinvestit les composantes métriques des poèmes pour tisser « une seconde peau ${ }^{1}$ » (Bick, 1968) qui vient pallier le défaut de contenance de la première peau psychique. La seconde peau métrique et poétique de Jonathan évoque la figure de la peau « oripeau » (Anzieu, 1985, p. 149) qui vient éblouir les autres membres du

1. E. Bick a proposé le modèle de la seconde peau pour qualifier des stratégies de substitution chez le nourrisson en vue de traverser les failles survenues lors de la constitution de la première peau. Au lieu de trouver à introjecter une peau contenante dans les premières relations à l'objet primaire, le nourrisson trouve un contenant de substitution dans sa propre musculature. 
groupe, protéger et asseoir le fantasme narcissique d'une peau commune jamais arrachée qui le confirme dans son omnipotence. Cette seconde peau et ses artifices attirent tous les regards dans le groupe mais privent Jonathan d'une parole sur ce qu'elle recouvre, à savoir les vécus d'agonies primitives que la lecture des textes permet d'identifier. Dans le groupe cependant, seuls les commentaires sur la maîtrise du style poétique fusent, faisant obstacle à une parole subjectivante témoignant d'une reconnaissance de la souffrance.

Dans le cas de Jonathan, la suspension du travail sur le noyau pulsionnel au bénéfice du renforcement de la couche externe des écrits a constitué un prérequis nécessaire pour qu'il puisse se maintenir dans le groupe et éprouver un sentiment de sécurité lui permettant de donner à voir peu à peu la violence qui l'habite derrière la maîtrise poétique. Voilà le passage qu'il insère dans une histoire collective où chaque participant du groupe est invité à contribuer :

«Participant 1 : Dans un mois c'est Noël. Heureusement que Noël est là, pour donner un peu de gaieté pendant cette période de froid.

Jonathan : Le père Noël, cette année-là, était en dépression et la dernière chose qu'il eut envie de faire était de sortir par un froid polaire sur ce foutu traîneau et distribuer des cadeaux à des chiards de plus en plus ingrats.

Participant 3 : Plutôt que son traîneau il prit sa moto-neige et se sentit plus en joie. Il allait et venait vers les enfants leur distribuer des friandises. L'atmosphère joyeuse de la journée inondait de bonheur tout ce petit monde. »

Cette intervention de Jonathan dans le texte collectif survient après une absence imprévue de la thérapeute et l'attaque du lien avec le groupe par les écrits témoigne de l'angoisse de la perte de ce lien. Cependant, l'écriture du texte collectif, et la survivance à la destructivité dont les autres interventions dans le texte témoignent, se constituent comme contenant et permettent à Jonathan d'éprouver la fiabilité du groupe. Vers la fin de notre période d'étude, Jonathan laissait entrevoir la possibilité d'investir le groupe, non plus seulement comme l'objet de l'actualisation traumatique, mais aussi comme un objet suffisamment bon et fiable pour accueillir les parts honteuses de son intériorité et engager le processus de transformation dans le partage avec le groupe.

«Essayer d'être un autre est une façon de devenir soi-même dans un regard neuf on récolte ce que l'on sème avec une approche différente l'autre se meut en conséquence on a beau rembobiner on ne trouve plus la séquence un instant dans la peau d'une autre chair 
ne se vend ni en boutique ni aux enchères on peut se rétracter et regagner son corps ou garder cette enveloppe pour un nouvel essor perdre à jamais ce contact qui dérive vers l'autre, ce sont des choses qui arrivent. »

Ces vers semblent témoigner de l'enjeu du groupe d'écriture pour Jonathan et du va-et-vient dans le rapport à cette enveloppe-écriture qu'il crée avec les autres : est-ce le support d'un enfermement sur soi-même ou une potentielle source de transformation? Les fonctions essentielles de cette enveloppe de protection, ici la seconde peau-poème, concernent la possibilité pour le sujet de se maintenir dans le monde et dans l'expérience de création, et de réduire la menace d'actualisation d'un vécu de rupture dans la rencontre avec l'environnement. Alors que pour Jonathan le dispositif du groupe a permis à son processus d'écriture de ne pas se figer dans sa fonction défensive, le danger de tels investissements réside dans l'édification d'une écriture rigide et impénétrable qui ne permettrait pas à son auteur le travail de mise en forme sur la couche interne de l'espace psychique. La rencontre avec les lecteurs risque alors de s'installer dans l'échec du partage de l'expérience et la répétition de vécus de rupture dans cette rencontre. L'environnement, pris entre l'éblouissement et l'aveuglement, ne peut accéder à la reconnaissance des éprouvés douloureux.

\section{MONTRER POUR MIEUX CACHER}

L'atelier d'écriture met à l'épreuve volontairement les limites du sujet, ce qui nous a permis de mettre en évidence l'investissement de l'écriture comme enveloppe de protection. Qu'en est-il de la place de ces investissements dans le cas de la création littéraire?

L'une des écrivaines interrogée utilise l'expression « amour/haine » pour qualifier sa relation à l'écriture. Son expérience de l'écriture est marquée par des vécus de discontinuité entre le temps d'écriture et le temps de la publication : "Au début j'aimais bien jouer avec les mots, maintenant j'aime la polysémie des mots [...] La forme et la manière dont on va exprimer une phrase, ça peut amener à différentes fins. La forme est très importante pour la direction de l'histoire. [...] Écrire permet de voir une autre logique au pouvoir, parce que finalement notre logique ici on la connaît parfaitement, on sait comment les choses fonctionnent, on sait qu'il y a des choses qui ne se font pas, qui ne se disent pas et qui ne pourront jamais se faire [...] c'est agréable je trouve d'avoir un autre monde. [...] C'est un effet jubilatoire de voir qu'on arrive à mettre en relation un événement avec un autre et que ça donne une histoire cohérente. C'est surtout cela qui me plaît dans l'écriture, 
c'est d'insérer des choses très hétéroclites et de voir que finalement on arrive à en faire une ligne qui est parfaitement logique. »

«On croit qu'une fois qu' on est publié, on y est arrivé, ça y est. Mais pas du tout, $c^{\prime}$ est à peine le commencement. [...] C'est déjà difficile de se faire éditer, on est tout content quand ça arrive, et on est sûre qu'après on va avoir des articles, être invitée à des lectures, avoir des retours, quelque chose d'un peu stimulant. Et pas du tout. [...] On n'a aucun retour sur rien, [...] C'est la partie otage complète du métier d'écrivain. Autant on est libre donc on pourrait écrire ce que l'on veut, autant après on ne peut plus rien faire. [...] L'écriture pure j'aime, et tout ce qui va à côté je déteste [...] on est libre d'écrire ce qu'on veut, de gérer son temps, et puis finalement on est complètement dépendant des autres et on n'a pas son mot à dire, pour rien [...] Quand on écrit on peut construire quelque chose, mais c'est l'avant et l'après qui sont plus difficile à gérer. [...] C'est tout ce qui est à côté de l'écriture qui a tendance à déprimer. Parce que des fois, c'est trop pénible. »

De manière générale, l'entretien mené avec l'écrivaine a laissé entrevoir une histoire marquée par des vécus d'impuissance. Dans les propos cités ci-dessus, elle témoigne de ce passage douloureux entre " l'effet jubilatoire » de l'écriture et la souffrance de l'absence de retour lors de la publication. L'effet jubilatoire émane de la satisfaction de construire, d'assembler, de mettre en relation des éléments pour réaliser un texte qui lui permet de voir " une autre logique au pouvoir ». Cependant, ces expériences de plaisir, qui s'ancrent presque exclusivement sur la manipulation de la langue, ne résistent pas au regard de l'autre. La recherche littéraire qui ne trouve pas à s'appuyer sur un interlocuteur se replie sur un travail de la matière qui vient contrecarrer les vécus d'impuissance. La création répond aux menaces de l'environnement en surinvestissant les défenses par retournement de la passivité en activité dans le maniement de la langue. Alors que l'œuvre choisie par l'auteure pour l'entretien figure des expériences de dénuement et de vide, tant dans son contenu que dans un style qu'elle qualifie ellemême de "squelettique ", le travail de la langue les enveloppe d'un excès de jeux de mots extrêmement maîtrisés qui donnent à vivre au lecteur le sentiment étrange de ne pas savoir s'il faut rire ou pleurer. L'enveloppe des mots cherche à neutraliser les vécus d'impuissance et de dénuement en les remplaçant par une maîtrise linguistique toute-puissante.

Ainsi, la création a partie liée avec un mouvement de retournement de la passivité en activité. Le sujet doit pouvoir se ressaisir des expériences projetées dans la matière pour entamer leur mise en forme en vue de l'intégration psychique. Cependant, le risque réside dans le surinvestissement 
de l'enveloppe externe toute-puissante. Dans un premier temps, ce type d'enveloppe permet bien au sujet d'accéder à une certaine reconnaissance, qui cependant ne résiste pas à l'actualisation des vécus d'impuissance lors de la rencontre avec l'environnement. L'écrivaine le dit bien : "On croit qu'une fois qu'on est publié, on y est arrivé, ça y est. Mais pas du tout, c'est à peine le commencement. »Ce commencement, on peut l'entendre comme la répétition d'une expérience de discontinuité dans la rencontre que donne à vivre le processus de publication. La création peut alors s'engager sur deux voies. Le sujet peut s'appuyer sur les fonctions protectrices de l'enveloppe défensive pour prendre le risque d'aller au-devant d'un partage des éprouvés internes avec le monde. Cette voie vers la symbolisation procède d'un continuel travail d'ajustement dans un va-et-vient entre une écriture qui protège et une écriture qui représente. La majorité des expériences étudiées ont témoigné de ce va-et-vient. Dans le processus de création littéraire, chaque œuvre vient marquer un temps dans cette hésitation entre le montrer et le cacher.

La seconde voie prise par le sujet soumis à l'actualisation traumatique est de se retirer derrière l'enveloppe défensive et de la renforcer au risque de creuser l'écart, toujours plus douloureux, entre soi et son environnement. Dans ce cas de figure, le sujet créateur lance un appel pour être vu et entendu, mais, paradoxalement, il s'applique à cacher ce qu'il souhaiterait voir être reconnu, dans une probable impossible confrontation à une souffrance extrême. On peut supposer, toutefois, que tant que le sujet continue à créer, le retrait n'est jamais total car chaque expérience de création, en répétant les vécus traumatiques, vient aussi réinterroger les voies privilégiées jusque-là et lancer un nouvel appel à une réponse de l'environnement. La possibilité d'une rencontre avec un interlocuteur qui pourra voir ce que les artifices cherchent à cacher, et supporter ce qu'il y trouvera, reste ouverte.

\section{CONCLUSION}

L'exploration du processus d'écriture dans les deux dispositifs étudiés nous a permis de mettre en évidence la place centrale des enjeux de continuité/ discontinuité dans la tentative de partage et de mise en sens par l'écriture d'expériences impensables. Ces enjeux se déploient autour du couple montrer/cacher et de la manière dont il guide le travail de mise en forme du texte en vue d'une présentation aux lecteurs. Ce travail donne lieu à différents procédés d'écriture qui cherchent à soutenir l'expression des éprouvés et leur reconnaissance par les interlocuteurs, tout en protégeant l'auteur de vécus d'intrusion. Ce processus de composition du texte se déploie notamment à partir des modalités sonores et rythmiques de l'écriture qui peuvent faire l'objet d'un investissement protecteur, défensif, et/ou symbolisant. 
La prégnance d'un surinvestissement défensif du style des écrits témoigne de la nécessité, pour le sujet, de matérialiser une enveloppe d'écriture externe suffisamment solide pour se maintenir dans le monde. L'enjeu des enveloppes d'écriture réside dans la rencontre avec un interlocuteur qui serait à même de repérer et de valider la fonction de réassurance narcissique qu'elle assurent, tout en pouvant reconnaître et attester de ce que les écrits cherchent à protéger, voire à cacher.

\section{BIBLIOGRAPHIE}

ANZIEU, D. 1978. Le groupe et l'inconscient, Paris, Dunod.

ANZIEU, D. 1981. Le corps de l'œuvre, Paris, Gallimard.

ANZIEU, D. 1985. Le Moi-peau, Paris, Dunod, 1995.

BICK, E. 1968. «The experience of the skin in early object-relations », The International Journal of Psychoanalysis, ${ }^{\circ} 49$, p. 484-486.

CADOUX, B. 2013. Écritures de la psychose, Vareilles, La Rumeur Libre.

CHOUvier, B. 1998. Symbolisation et processus de création, Paris, Dunod.

CHOUviER, B. ; BRUN, A. 2011. Les enjeux psychopathologiques de l'acte créateur, Bruxelles, De Boeck.

EMMANUELLI, M. 2001. « Place et rôle de l'affect dans les processus de sublimation artistique », Bulletin de psychologie, $\mathrm{n}^{\circ}$ 54, p. 553-561.

FreUD, S. 1925. « Note sur le "Bloc-notes magique" », dans CEuvres complètes, XVII, Paris, Puf, 1992.

Guillaumin, J. 1980. « La peau du centaure. Le retournement projectif de l'intérieur du corps dans la création littéraire », dans Corps création. Entre lettres et psychanalyse, Presses universitaires de Lyon, p. 227-267.

Guillaumin, J. 1983. "La souffrance travaillée par la pensée dans l'écriture », dans J. Caïn, C. David, M. Faïn, J. Guillaumin, S. Melor Picaut, M. Olender, Souffrance, plaisir et pensée, Paris, Les Belles Lettres, p. 61-131.

Guillaumin, J. 1998. " Le jugement esthétique, un instrument logique étrange entre l'intime et l'universel », dans B. Chouvier Symbolisation et processus de création, Paris, Dunod, p. 35-56.

JACQUES, A. ; LEFEBVRE, A. 2005. « La création artistique....un en deçà du désir », Cahiers de psychologie clinique, $\mathrm{n}^{\circ} 24, \mathrm{p} .187-213$.

JANIN, C. 2005. «Au cœur de la théorie psychanalytique : le traumatisme», dans F. Brette, M. Emmanuelli, G. Pragier, Le traumatisme psychique. Organisation et désorganisation, Paris, Puf, p. 43-55.

LEMPEN, O. 2014. Processus et fonctions psychiques de l'écriture : de la création littéraire au groupe à médiation, thèse de doctorat sous la direction du professeur Pascal Roman, université de Lausanne, Suisse.

M'UZAN, M. DE. 1964. «Aperçus sur le processus de la création littéraire », dans De l'art à la mort, Paris, Gallimard, 1977, p. 3-27.

Roman, P. 2007. "Figures du négatif et "expérience" créatrice », dans A. Brun, J.-M. Talpin, Cliniques de la création, Bruxelles, De Boeck, p. 117-140. 
RoussiLlon, R. 1998. « Désir de créer, besoin de créer, contrainte à créer, capacité de créer », dans B. Chouvier, Symbolisation et processus de création, Paris, Dunod, p. 158-171.

STERN, D.N. 1985. Le monde interpersonnel du nourrisson, Paris, Puf, 1989.

WIDLÖCHER, D. 1999. «La méthode du cas unique », dans F. Villa, P. Fédida, Le cas en controverse, Paris, Puf, p. 191-200.

WinNiCOTT, D.W. 1971. Jeu et réalité, Paris, Gallimard, 1975.

\section{Résumé}

Les auteurs présentent des réflexions issues d'une recherche ayant pour objet l'étude des processus d'écriture d'écrivains publiés et de participants à un atelier d'écriture en centre de jour. Cette double approche de l'écriture comme expérience créatrice permet d'explorer trois temps du processus : la mise à l'écriture ; le travail de la matière ; le partage avec les lecteurs. La mise à l'écriture s'appuie sur la mobilisation des premiers accordages affectifs dans leurs modalités rythmique et sonore alors que la composition du texte a pour enjeu le travail des limites dedans/dehors en vue du partage avec des lecteurs. Ces expériences de création montrent que l'écriture peut avoir une fonction d'enveloppe protectrice qui soutient le sujet dans sa rencontre avec un interlocuteur, permettant un travail de symbolisation.

Mots-clés

Processus de création, écriture, limites psychiques, enveloppes psychiques, création littéraire, atelier d'écriture.

WORK ON PSYCHIC LIMITS IN THE PROCESS OF WRITING.

EXPLORATION IN THE LITERARY CREATION AND IN A WRITING WORKSHOP

\section{Abstract}

The authors present reflections resulting from a research that focussed on the process of writing in two areas: the literary creation of published writers and the writing of participants who took part to a group in an adult day centre. The joint analysis of these writing experiences leads to the exploration of three successive aspects of the process: tackling writing; working on the material; sharing with readers. Tackling writing relies on mobilizing the first affect attunements in their rhythmic and sonorous aspects, while shaping the text challenges the work on inner and outer psychic limits in view of sharing with readers. These creative experiences show that writing can act as a protective envelope that supports the subject in the encounter with an interlocutor, thus enabling a process of symbolization.

Keywords

Creative process, writing, psychic limits, psychic envelopes, literary creation, writing workshop. 